Erratum

\title{
On the Reduction of Holt's Problem
}

\section{to a Finite Interval}

Katalin Balla and Mária Vicsek

Computer and Automation Institute, Hungarian Academy of Sciences,

Victor Hugo u. 18-22, H-1132 Budapest, Hungary

Numer. Math. 51, 291-302 (1987)

The correct expression that should stand just after formula (1.3) in the next line, p. 291 is

$$
p(t)=t^{2}\left(p_{0}+p_{1} / t^{2}\right)
$$

